\title{
Comparative proteomic analysis to identify the novel target gene of angiotensin II in adrenocortical H295R cells
}

\author{
Ryo Ito ${ }^{1)}$, Hiroki Shima ${ }^{2}$, Koji Masuda ${ }^{3)}$, Ikuko Sato ${ }^{1)}$, Hiroki Shimada ${ }^{1)}$, Atsushi Yokoyama ${ }^{1}$, \\ Katsuhiko Shirahige ${ }^{3)}$, Kazuhiko Igarashi ${ }^{2)}$ and Akira Sugawara ${ }^{1)}$ \\ 1) Department of Molecular Endocrinology, Tohoku University Graduate School of Medicine, Sendai 980-8575, Japan
2) Department of Biochemistry, Tohoku University Graduate School of Medicine, Sendai 980-8575, Japan
3) Research Center for Epigenetic Disease, Institute of Molecular and Cellular Biosciences, The University of Tokyo, Tokyo 113-0032,
Japan
}

\begin{abstract}
Angiotensin II (Ang II) is a well-known peptide that maintains the balance of electrolytes in the higher vertebrates. Ang II stimulation in the adrenal gland induces the synthesis of mineralocorticoids, mainly aldosterone, through the upregulation of aldosterone synthase (CYP11B2) gene expression. Additionally, it has been reported that Ang II activates multiple signaling pathways such as mitogen-activated protein kinase (MAPK) and $\mathrm{Ca}^{2+}$ signaling. Although Ang II has various effects on the cellular signaling in the adrenal cells, its biological significance, except for the aldosterone synthesis, is still unclear. In this study, we attempted to search the novel target gene(s) of Ang II in the human adrenal H295R cells using a proteomic approach combined with stable isotopic labeling using amino acid in cell culture (SILAC). Interestingly, we found that Ang II stimulation elevated the expression of phosphofructokinase type platelet (PFKP) in both protein and mRNA levels. Moreover, transactivation of PFKP by Ang II was dependent on extracellular-signal-regulated kinase (ERK) 1/2 activation. Finally, we observed that Ang II treatment facilitated glucose uptake in the H295R cells. Taken together, we here identified PFKP as a novel target gene of Ang II, indicating that Ang II not only stimulates steroidogenesis but also affects glucose metabolism.
\end{abstract}

Key words: Angiotensin II, Adrenocortical cell, Proteome analysis, Phosphofructokinase type platelet

\begin{abstract}
ANGIOTENSIN II (ANG II) is a bioactive peptide, and is essential for the maintenance of the balance of electrolytes in the higher vertebrates [1]. The secretion of this peptide is stringently controlled by several enzymes, such as renin and angiotensin-converting enzyme (ACE) [2]. In the adrenal gland, Ang II stimulates zona glomerulosa which is the external layer of the adrenal cortex and facilitates the transcription of steroidogenic enzymes including steroid 21-hydroxylase (CYP21A) [3], $3 \beta$-hydroxysteroid dehydrogenase (HSD3 $\beta$ ) [4], and aldosterone synthase (CYP11B2) [5]. These enzymes synthesize aldosterone, a major mineralocorticoid, that enables the kidney to reabsorb salt and water [6]. Through these sequential reactions, named as the renin-

Submitted Mar. 14, 2020; Accepted Nov. 11, 2020 as EJ20-0144 Released online in J-STAGE as advance publication Dec. 26, 2020 Correspondence to: Akira Sugawara, MD, PhD, Department of Molecular Endocrinology, Tohoku University Graduate School of Medicine, 2-1 Seiryo-machi, Aoba-ku, Sendai 980-8575, Japan. E-mail: akiras2i@med.tohoku.ac.jp
\end{abstract}

angiotensin-aldosterone system (RAAS), Ang II plays an important role in the mineral homeostasis.

In the adrenal zona glomerulosa, Ang II stimulates various signaling pathways via the Ang II type I receptor (AT1R) that belongs to the $\mathrm{G}$ protein-coupled receptor (GPCR) family [7]. Like other GPCRs, AT1R activates inositol triphosphate (IP3) signaling and releases $\mathrm{Ca}^{2+}$ from the endoplasmic reticulum. Then, the cytosolic $\mathrm{Ca}^{2+}$ activates $\mathrm{Ca}^{2+} /$ calmodulin-dependent protein kinase $(\mathrm{CaMK})$ and protein kinase $\mathrm{C}$ (PKC) [8]. Moreover, AT1R also activates mitogen-activated protein kinase (MAPK) signaling, such as extracellular-signal-regulated kinase (ERK) [9] and p38 signaling [10]. Therefore, Ang II can activate multiple cellular signaling pathways. However, the biological significance of Ang II in the adrenal gland remains poorly understood except for mineralocorticoid synthesis.

Proteome analysis using mass spectrometry is a powerful tool for the comprehensive analysis of gene expression. Several studies have been carried out to 
search for the target genes of Ang II in H295R cells derived from human adrenocortical tumor by microarray analysis $[11,12]$. However, there remains a possibility that the analysis of mRNA expression does not reflect the actual gene expression since they are not only controlled by transcriptional efficiency and mRNA stability but also by translational efficiency and protein stability. With regards to these aspects, proteomic analysis is a method to directly measure gene expression. Moreover, the recent development of mass spectrometry technology combined with isotopic labeling of proteins (SILAC) can perform a more quantitative comparison of protein expression [13]. In the present study, we explored the novel target gene of Ang II in the adrenal cortical cells using SILAC-based proteomic analyses to define the unknown effect of Ang II in the adrenal gland.

\section{Materials and Methods}

\section{Cell culture}

Human H295R cells were cultured in a DMEM/Ham F-12 medium (Fujifilm Wako Pure Chemical, Osaka, Japan) including $4.18 \mathrm{mM}$ potassium chloride supplemented with $10 \%$ fetal bovine serum (FBS), insulintransferrin-selenium-G (ITS) supplements (Thermo Fisher Scientific, Pittsburgh, PA, USA), $1.25 \mathrm{mg} / \mathrm{mL}$ of BSA (Sigma-Aldrich St Louis, MO, USA), $5.35 \mu \mathrm{g} / \mathrm{mL}$ of linoleic acid (LA, Sigma-Aldrich), $100 \mathrm{U} / \mathrm{mL}$ of penicillin, and $100 \mu \mathrm{g} / \mathrm{mL}$ of streptomycin. This cell line was grown at $37^{\circ} \mathrm{C}$ in $5 \% \mathrm{CO}_{2}$. For Ang II stimulation, H295R cells were incubated in DMEM (Fujifilm Wako) containing $1 \%$ charcoal-treated FBS and antibiotics with angiotensin II (Sigma-Aldrich).

\section{Stable isotope labeling using amino acids in cell culture (SILAC)-based quantitative proteomic analysis}

According to a previous report [14], H295R cells were cultured in RPMI media (Thermo Fisher Scientific) supplemented with $10 \%$ dialyzed FBS, ITS, BSA, LA, and antibiotics. The medium was also supplemented with normal lysine and arginine to create a "light" medium in one culture, or ${ }^{13} \mathrm{C} 6,{ }^{15} \mathrm{~N}$-lysine and ${ }^{13} \mathrm{C} 6$-arginine (Fujifilm Wako) to create a "heavy" medium in another culture. After the cells were cultured in these media for 3 weeks, the cells that were cultured in the heavy medium were treated with $100 \mathrm{nM}$ Ang II, whereas the cells in light medium were not treated. For the preparation of cell extracts, the collected cells that were cultured in heavy/ light conditions were mixed in the same tube, lysed by sonication in TNE buffer $(20 \mathrm{mM}$ Tris- $\mathrm{HCl}, 137 \mathrm{mM}$ $\mathrm{NaCl}, 2$ mM EDTA, 1\% NP-40, and protease inhibitor cocktail III (pH 7.9) (Merck Millipore, Burlington, MA,
USA), and the debris was removed by centrifugation. These extracts were subjected to SDS-polyacrylamide gel electrophoresis (SDS-PAGE) and visualized by colloidal blue staining (Thermo Fisher Scientific). Each sample was dissected into approximately 30 sections, and subjected to a trypsin in-gel digestion procedure, as previously described [15]. The spectrum of the digested peptides was measured by nano LC-ESI-MS/MS using an LTQ Velos Orbitrap ETD instrument (Thermo Fisher Scientific). The spectral data were processed by a Mascot search (MATRIX SCIENCE, London, UK) to identify proteins, and by Proteome Discoverer (Thermo Fisher Scientific) to calculate the protein expression ratio. We excluded the data that is not satisfied with the false discovery rate (FDR) of peptide $<0.01$ and the number of peptide spectrum match (PSM) of protein $\geq 2$.

\section{Computational clustering and gene ontology (GO) analysis}

The protein expression ratio was analyzed by Perseus software [16] to determine the differentially expressed proteins $(p<0.01)$. The differentially expressed protein lists by Ang II treatment for 6, 12, or 24 hours were merged to define 161 proteins that altered the expression in at least one condition. For the 161 proteins, pair-wise Pearson correlation coefficients of expression ratios were computed for 6,12 , and 24 hours treatments. The distant matrix was defined as 1-Pearson correlation coefficients, which ranged from 0 to 2 when the pair gave perfect or inversely perfect correlation, respectively. Hierarchical clustering was performed using Ward's method of $\mathrm{R}$ and gplots package (R.C. team, R: A Language and Environment for Statistical Computing, https://www.r-project.org/, 2015., G. Warnes, B. Bolker, L. Bonebakker, R. Gentleman, W. Huber, A. Liaw, T. Lumley, M. Maechler, A. Magnusson, S. Moeller, M. Schwartz, and B. Venables, gplots: Various R Programming Tools for Plotting Data. (2015)). GO analysis was carried out using the DAVID bioinformatics suite (version 6.7) [17].

\section{Western blotting and antibodies}

Western blotting analysis was carried out using $\alpha$ PFKP (Cell Signaling Technology, CST, Danvers, MA, USA), $\alpha$-actin (Santa Cruz Biotechnology, Dallas, TX, USA), $\alpha$-ERK1/2 (CST), and $\alpha$-phospho ERK1/2 (CST). Densitometric analysis was obtained by Image J software.

\section{RNA isolation, complementary DNA (cDNA) synthesis, polymerase chain reaction (PCR), and statistics}

RNA isolation, cDNA synthesis and PCR were conducted as previously described [15]. The primer sequences for the human genes were as follows: 1) PFKP, 
forward, 5'-CTACAAGCGACTTGCCATCA-3', and reverse, 5'-ATCATAGATGGCGAGCATCC-3', 2) phosphofructokinase type liver (PFKL), forward, 5'-CCCTTT CAACATCCACGACT-3', and reverse, 5'-TCCCATAGT TCCGGTCAAAG-3', 3) phosphofructokinase type muscle (PFKM), forward, 5'-CCCGTGTCTTCTTTGTCC AT-3', and reverse, 5'-GTTGTAGGCAGCTCGGAGT C-3', 4) GAPDH, forward, 5'-ATCCCATCACCATCTTC CAG-3', and reverse, 5'-ATGAGTCCTTCCACGATAC C-3'. All values are expressed as the mean \pm standard deviation from three independent experiments. We used a two-tailed Student's $t$ test to analyze the differences between two groups.

\section{Measurement of glucose concentration of cultured medium}

H295R cells were cultured in DMEM/Ham's F-12 medium for 4 days and the medium was exchanged into DMEM containing $4.5 \mathrm{~g} / \mathrm{L}$ of glucose with/without 100 nM Ang II. After 36 and 72 hours of Ang II stimulation, the cultured medium was collected and the number of cells in each culture was measured by a hemocytometer. The glucose concentration in the incubated media was measured by a Glucose (HK) Assay Kit (Sigma-Aldrich).

\section{Results}

\section{Proteomic analysis to identify the novel target genes of angiotensin II}

In the adrenal gland, it is well known that Ang II stimulates various signaling pathways such as $\mathrm{Ca}^{2+} / \mathrm{CaMK}$ and MAPK signaling as well as governing aldosterone synthesis $[7,18]$. However, less is known about the other functions of Ang II in the adrenocortical cells. Therefore, we attempted to survey the novel target genes of Ang II with SILAC-based quantitative proteomic analysis in the present study. The cells cultured in the "heavy" labeled medium were stimulated by $100 \mathrm{nM}$ Ang II for 6,12 , or 24 hours and the cells were combined with the control cells, which were harvested in the "light" medium without Ang II. Then, the extracts derived from these cells were subjected to SDS-PAGE and mass spectrometric analysis (Fig. 1A). The results show that 3,464, 4,145, and 4,653 proteins were identified and satisfied our criteria (see Materials and methods) in 6, 12, and 24 hours samples, respectively (Fig. 1B). These experiments revealed the existence of unknown Ang II target genes in addition to the genes already known as an Ang II target such as RHOB (Table 1) [11].

Furthermore, clustering analysis divided the proteins into the four groups, which altered the expression with a statistically significance difference $(p<0.01)$ (Fig. 1C). We focused on cluster 3 whose expression increased depending on the treatment time (Fig. 1D). This cluster was also subjected to the gene ontology (GO) analysis. $\mathrm{GO}$ analysis revealed that the proteins grouped as cluster 3 were related to the "fructose 1,6-bisphosphate metabolic process $(p=0.013)$ " and the "fructose metabolic process $(p=0.042)$ " (Fig. 1E). Fructose metabolism plays a major role in glucose metabolism, particularly in glycolysis [19]. Therefore, we examined the expression changes that were focused on the proteins related to glycolysis (Table 2A). After 24 hours from Ang II stimulation, the expression of several glycolysis-related proteins such as HK1, HK2 GPI, TPI, ENO1 were slightly increased (about 1.2-fold) and PFKP and ALDOC expressions were especially up-regulated with a statistically significance difference (1.52-fold and 1.34-fold, respectively). Compared to this result, the expressions of pentose phosphate pathway (PPP)-related proteins did not change (Table 2B). These results suggested that Ang II stimulation did not increase the PPP-related proteins, but specifically raised the glycolysis-related proteins PFKP and ALDOC.

\section{PFKP is a novel Ang II target gene in the protein/ mRNA levels}

We validated the incremental PFKP expression by Ang II treatment. Western blotting combined with densitometric analysis showed a 1.74-fold upregulation of PFKP, while the expression of actin changed very little (1.07-fold) (Fig. 2A). Three independent experiments indicated that the PFKP/actin ratio increased 1.68-fold (Fig. 2B). This agreed with the mass spectrometry analysis (Table 2). Next, we examined whether this PFKP expression change was in protein or mRNA levels. RTqPCR analysis showed an incremental change in PFKP mRNA expression at 6,12 , and 24 hours treatment (Fig. 2C). The phosphofructokinase family is composed of PFKP (platelet type), PFKM (muscle type), and PFKL (liver type). Hence, we investigated the alteration of PFKM and PFKL mRNA expression levels. These results showed that the mRNA expression levels of PFKM and PFKL were not increased by Ang II stimulation (Fig. 2D and 2E), which were in agreement with mass spectrometry analysis (Table 2). Taken together, we considered that PFKP is a novel Ang II-response gene both in mRNA- and protein-levels. We next examined whether $1 \%$ charcoal-treated FBS affected PFKP mRNA expression by Ang II stimulation. As shown in Fig. 2F, Ang II-stimulated PFKP mRNA expression level in the presence of $1 \%$ charcoal-treated FBS was identical to that in the absence of FBS, indicating that $1 \%$ charcoaltreated FBS did not affect it. 
a

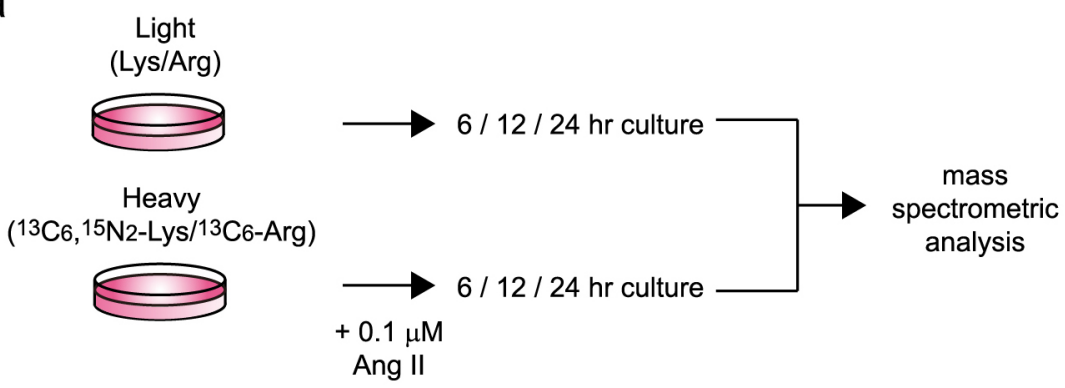

b
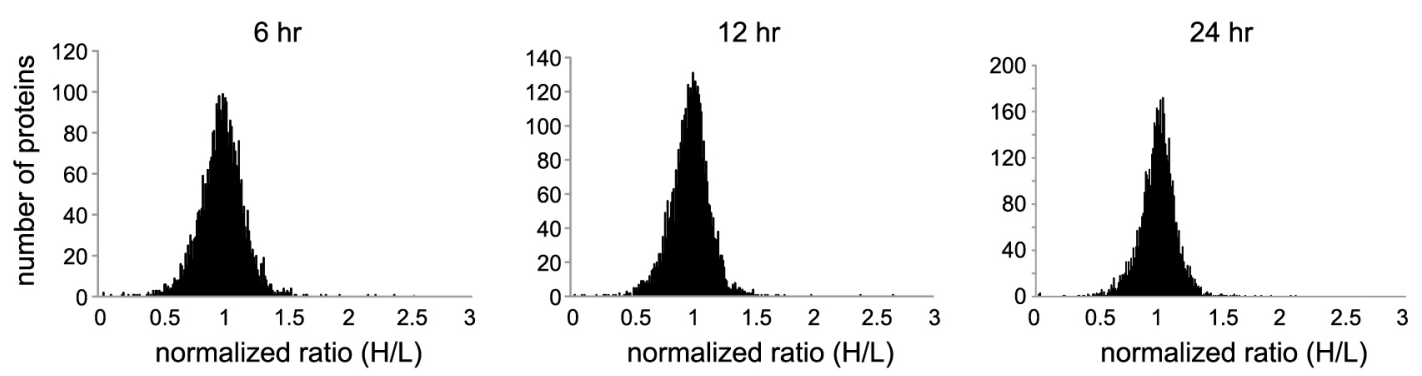

C
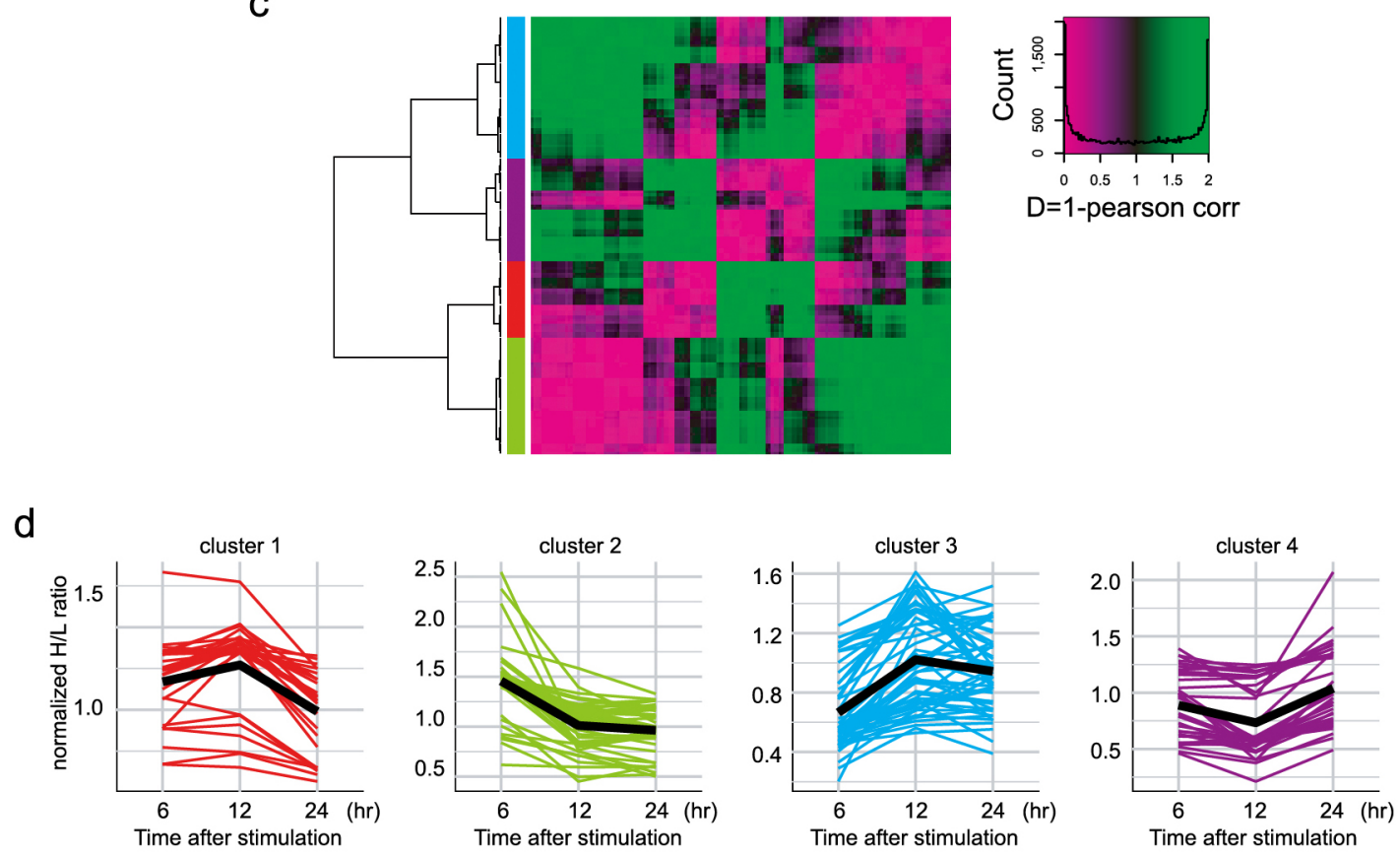

e

\begin{tabular}{|c|c|}
\hline GO term & $P$ value \\
\hline fructose 1,6-bisphosphate metabolic process & 0.013 \\
\hline macromolecular complex assembly & 0.024 \\
\hline macromolecular complex subunit organization & 0.031 \\
\hline fructose metabolic process & 0.042 \\
\hline Golgi vesicle transport & 0.043 \\
\hline
\end{tabular}

Fig. 1 Comprehensive proteome analysis using LC-MS/MS with SILAC to search the Ang II-targeted proteins.

(A) Experimental scheme for quantitative proteomics. (B) Histograms of the number of the protein expression ratio (heavy/light, described as H/L) at 6,12 and 24 hours from $0.1 \mu \mathrm{M}$ Ang II stimulation. (C) Hierarchical cluster of 161 proteins which changed their expression levels at least one time point. (D) The alteration of the expressions divided into 4 clusters. The average of the expression changes was described as a bold line in the respective groups. (E) The results of GO analysis of cluster 3. 
Table 1

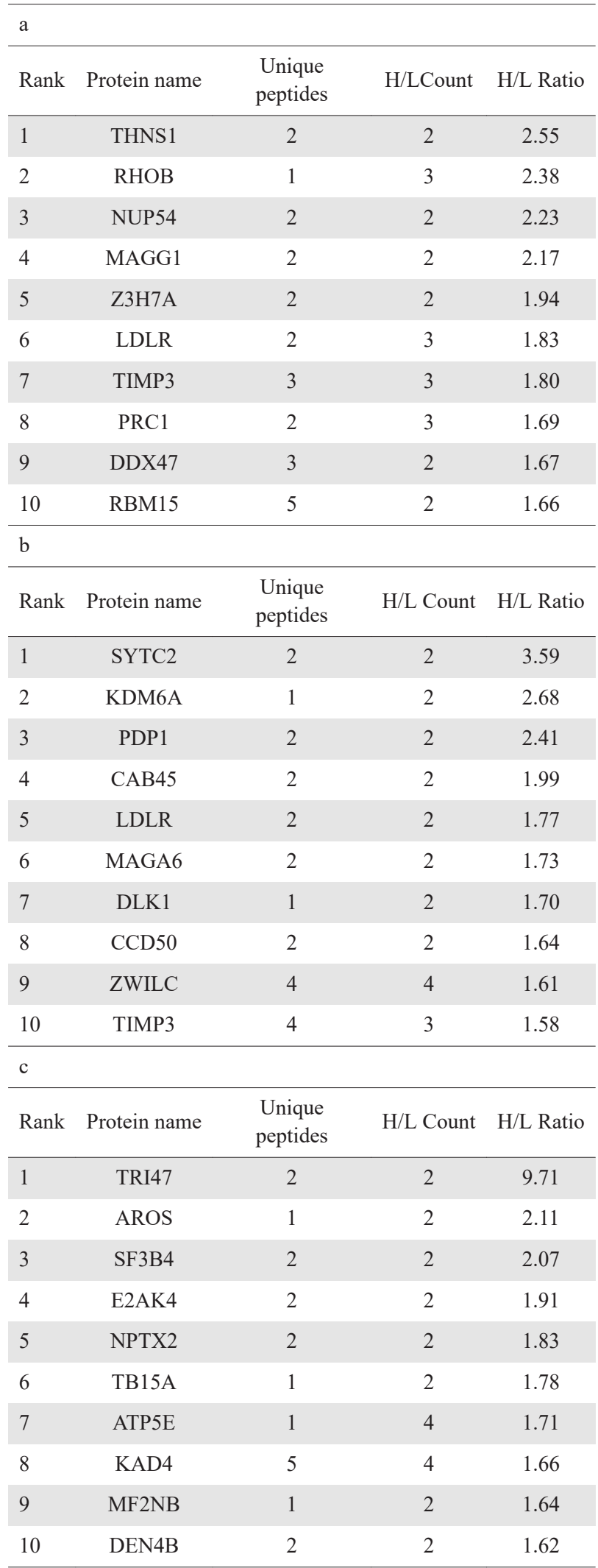

Top 10 proteins which increased their expression by Ang II stimulation at respective time point.

The proteins are listed in order from 1 to 10 according to their increased expression ratio each 6 (a), 12 (b) and 24 (c) hours from Ang II stimulation. "H/L Count" means the number of spectra to calculate H/L Ratio.

\section{Ang II response of PFKP was dependent on MEK/ERK signaling}

In the adrenal gland, Ang II activated several signaling pathways via its receptor, AT1R. So far, classical MAPK, p38 MAPK, and $\mathrm{Ca}^{2+} / \mathrm{CaMK}$ signaling were reported to be activated by Ang II stimulation in H295R cells [18]. Then, we attempted to reveal the signaling cascades involved in the upregulated expression of PFKP by Ang II. The PFKP upregulation responding to Ang II was critically repressed with U-0126, which is an inhibitor of classical MAPK signaling via MEK/ERK, while the p38 MAPK signaling inhibitor SB203580 and CaMK2 inhibitor KN-93 did not suppress the expression (Fig. 3A and 3B). These results suggested that Ang II stimulation increased the PFKP mRNA through MEK/ERK signaling.

\section{Ang II affected the glucose intake in the adrenal cells}

As shown in this paper, we identified the PFKP as a new response gene of Ang II (Table 2 and Fig. 2). PFKP is a member of the phosphofructokinase family which catalyzes the phosphorylation of fructose-6-phosphate to fructose-1,6-bisphospate [20]. This reaction is considered as a rate-limiting step in glycolysis [21]. Therefore, we postulated that Ang II stimulation influenced glucose metabolism. We therefore examined the glucose concentration of the cultured medium in H295R cells. The concentration of glucose was significantly decreased in the medium after the cells were cultured with Ang II stimulation for 72 hours (Fig. 4A). Next, we checked the effect of Ang II on cell growth to calculate the glucose uptake per total cell number. A previous report showed that Ang II stimulated the cell cycle into progression during short-term treatment in H295R cells [22]. However, in our experiments, cell growth had a tendency to be slightly suppressed by Ang II treatment for 36 and 72 hours, though there was no statistical significance (Fig. 4B). Taken together, by calculating the glucose uptake per total cell number, we confirmed that Ang II stimulation increased the amount of glucose uptake (Fig. 4C).

\section{Discussion}

Although Ang II is well known as a bioactive peptide to induce aldosterone synthesis, little is known about its other effects on the adrenal gland. Previous studies investigated the target genes of Ang II in the adrenal cortical H295R cell line or primary cultured adrenal cells using microarray analysis [11, 12]. These reports focused on the early response genes belonging to transcription factors and the other target genes of Ang II remain elusive especially for more than 12 hours treatment. In this 
Table 2

\begin{tabular}{|c|c|c|c|c|c|c|}
\hline \multicolumn{7}{|l|}{$\mathrm{a}$} \\
\hline \multirow{2}{*}{ Protein name } & \multicolumn{2}{|c|}{$6 \mathrm{hr}$} & \multicolumn{2}{|c|}{$12 \mathrm{hr}$} & \multicolumn{2}{|c|}{$24 \mathrm{hr}$} \\
\hline & H/L Count & H/L Ratio & H/L Count & H/L Ratio & H/L Count & $\mathrm{H} / \mathrm{L}$ Ratio \\
\hline HK1 & 29 & 1.16 & 54 & 1.16 & 38 & 1.23 \\
\hline HK2 & 7 & 0.91 & 21 & $1.27^{*}$ & 21 & 1.22 \\
\hline GPI & 34 & 1.00 & 48 & 1.14 & 101 & 1.21 \\
\hline PFKP & 24 & 0.87 & 25 & $1.34 * *$ & 40 & $1.52 * *$ \\
\hline PFKM & 43 & 1.08 & 44 & 1.13 & 82 & 1.13 \\
\hline PFKL & 49 & 0.84 & 46 & 1.08 & 87 & 1.19 \\
\hline ALDOA & 38 & 0.95 & 55 & 1.09 & 68 & 1.12 \\
\hline ALDOB & \multicolumn{2}{|c|}{ N. D. } & 2 & 1.07 & \multicolumn{2}{|c|}{ N. D. } \\
\hline ALDOC & 24 & 0.76 & 42 & 0.96 & 36 & $1.34 * *$ \\
\hline TPI1 & 53 & 1.00 & 75 & 1.18 & 114 & $1.24 *$ \\
\hline GAPDH & 175 & 0.98 & 325 & 1.07 & 295 & 1.05 \\
\hline PGK1 & 35 & 0.83 & 74 & 1.14 & 103 & 1.18 \\
\hline PGAM1 & 26 & 0.74 & 33 & 0.97 & 32 & 0.96 \\
\hline PGAM5 & 11 & 1.03 & 13 & 0.97 & 2 & 0.70 \\
\hline ENO1 & 30 & 0.90 & 27 & 1.11 & 57 & 1.23 \\
\hline ENO2 & 3 & 0.68 & \multicolumn{2}{|c|}{ N. D. } & 5 & 0.93 \\
\hline ENO3 & \multicolumn{2}{|c|}{ N. D. } & 2 & 0.89 & 23 & 1.07 \\
\hline PKM & 407 & 0.91 & 587 & 0.99 & 824 & 1.09 \\
\hline \multicolumn{7}{|l|}{$\mathrm{b}$} \\
\hline \multirow{2}{*}{ Protein name } & \multicolumn{2}{|c|}{$6 \mathrm{hr}$} & \multicolumn{2}{|c|}{$12 \mathrm{hr}$} & \multicolumn{2}{|c|}{$24 \mathrm{hr}$} \\
\hline & H/L Count & H/L Ratio & H/L Count & H/L Ratio & H/L Count & H/L Ratio \\
\hline G6PD1 & 29 & 1.15 & 43 & 1.19 & 76 & 1.11 \\
\hline PGLS & 17 & 0.98 & 16 & 1.13 & 13 & 1.10 \\
\hline PGD & 13 & 0.99 & 14 & 1.02 & 33 & 0.97 \\
\hline RPIA & \multicolumn{2}{|c|}{ N. D. } & 2 & 0.78 & 3 & 0.84 \\
\hline RPE & \multicolumn{2}{|c|}{ N. D. } & 2 & 1.06 & 2 & 1.20 \\
\hline TKT & 71 & 0.97 & 77 & 0.97 & 147 & 0.95 \\
\hline TALDO1 & 30 & 1.03 & 19 & 1.07 & 21 & 1.08 \\
\hline
\end{tabular}

The alteration of the protein's expression related to glycolysis (a) or PPP (b). Asterisks indicate the increase of the expression with significant differences. $* p<0.05 ; * *<<0.01$. N.D., not detected.

study, we attempted to reveal novel targets of Ang II in adrenal H295R cells using proteomic analysis. H295R cells are derived from a human adrenocortical carcinoma, and are well known to preserve hormonal responses to Ang II and potassium [23]. Our results of SILAC-based quantitative proteomic analysis identified more than three thousand proteins that satisfied our criteria in each sample (Fig. 1B). For investigation of the long-span effect of Ang II treatment, we selected cluster 3 which increased the expression over time and they were sub- jected to GO analysis. The result of GO analysis indicated that the proteins including cluster 3 were involved in fructose metabolism which is a part of the glycolysis pathway (Fig. 1E, Table 2A). Taken together, we focused on and analyzed the expression changes of the PFKP protein responding to Ang II stimulation.

Analysis of PFKP mRNA expression showed that the increase of the PFKP protein by Ang II stimulation was due to transcriptional activation (Fig. 2C). So far, several studies have investigated the mechanism of transcrip- 
a

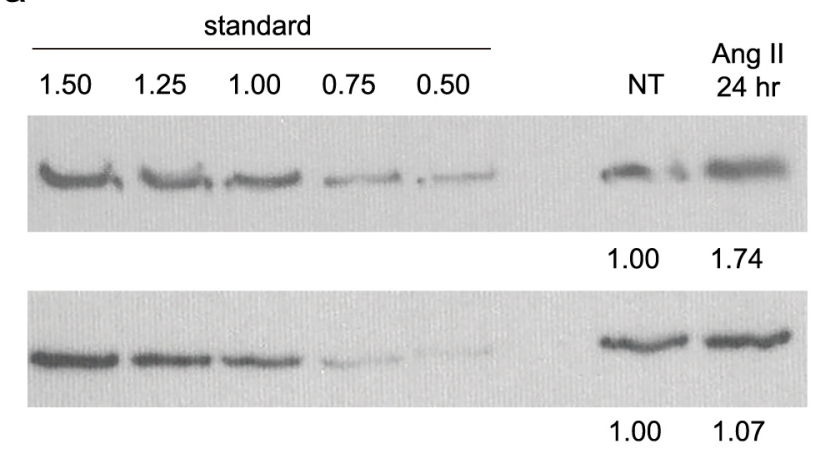

b

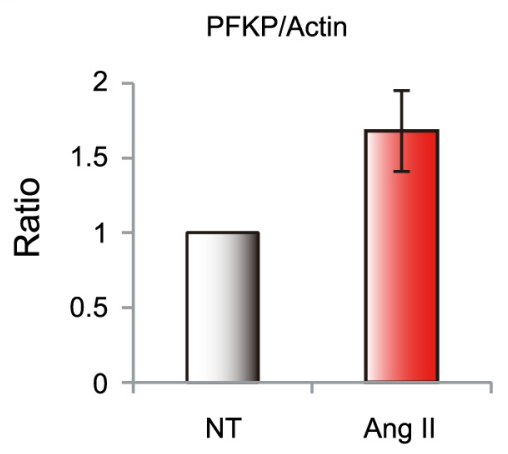

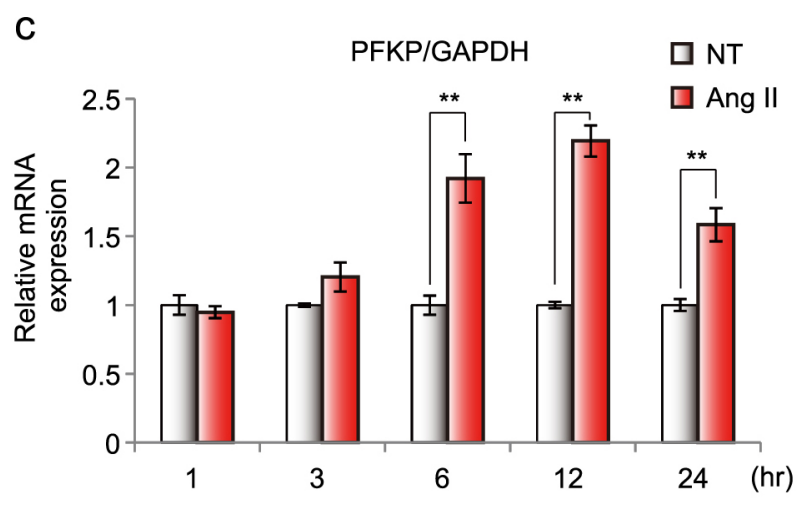

WB: $\alpha$-PFKP

WB: $\alpha$-Actin

d
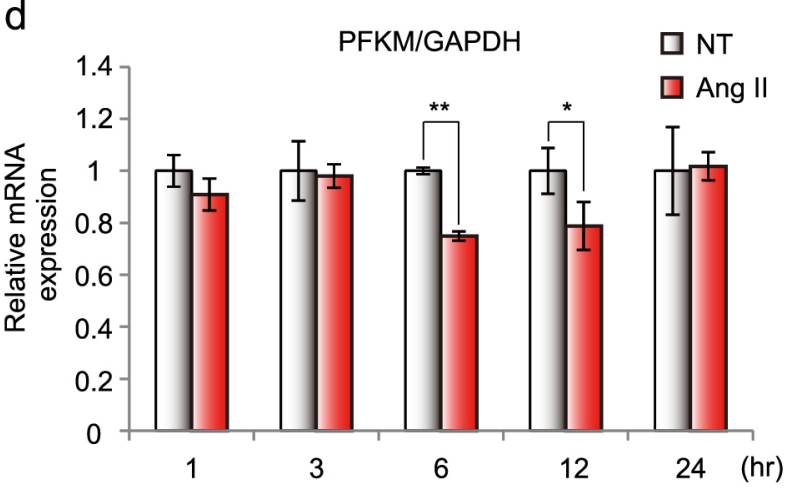

f
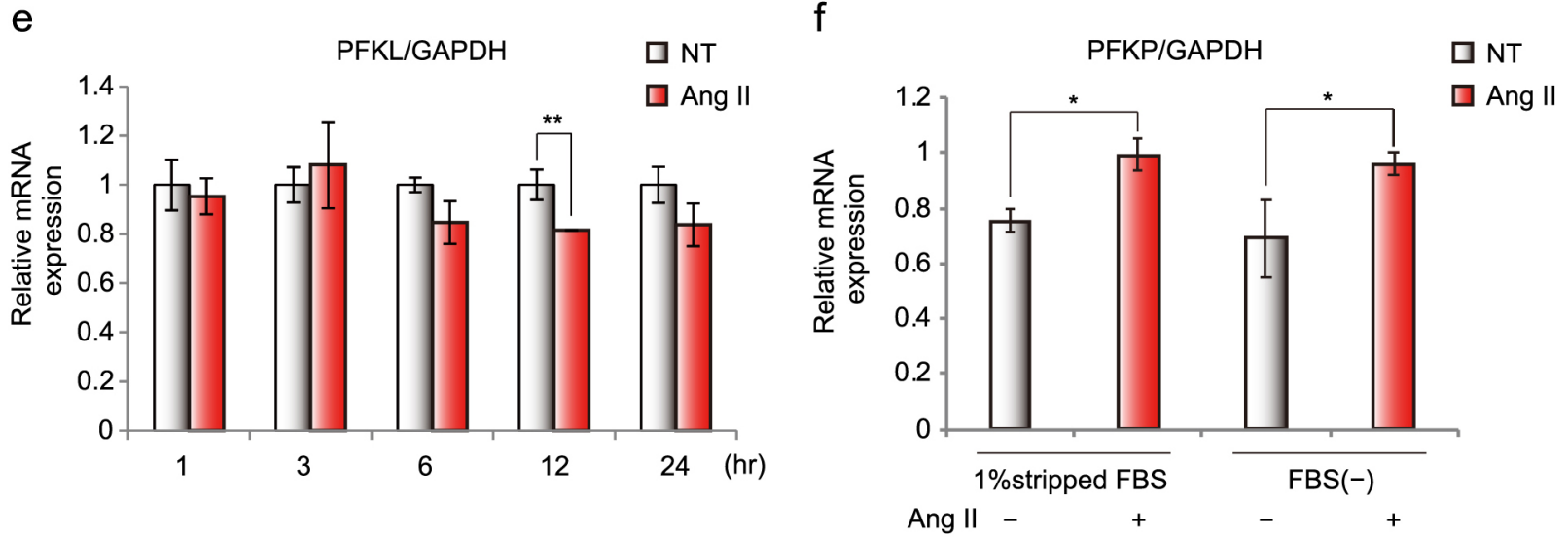

Fig. 2 Ang II raised the expression of PFKP in protein and $m R N A$ levels.

(A) The validation of the alteration of PFKP expression by western blotting. The numbers under each panel indicated the expression ratio measured by Image J. (B) Densitometric analysis was carried out based on the results from three independent experiments as in (A). (C-E) RT-qPCR was conducted using the primers for PFKP (C), PFKM (D), PFKL (E) and for GAPDH as a control. (F) RT-qPCR was conducted under the condition of H295R cells were stimulated by Ang II either with 1\% stripped FBS or without FBS. RT-qPCR intensities were normalized to GAPDH expression. Data are represented as means \pm standard deviations $(n=3)$, ratio to non-treated (NT, without Ang II) groups. $* p<0.05 ; * * p 0.01$.

tional regulation of the PFKP gene. Previous work showed that Krüppel-like factor 4 (KLF4) directly bound to the promoter of PFKP and activated its transcription [24]. More recently, transcriptional repressor ZBTB7A was reported to reduce the expression of PFKP in vivo and in vitro [25]. In this study, we found that MEK inhibitor U-0126 significantly repressed the up-regulation of PFKP mRNA by Ang II stimulation (Fig. 3A). MEK inhibition reduced ERK1/2 phosphorylation (Fig. 3B) and led to a decrease in the phosphorylation and activation of ERK1/2-targeted transcription factors [26]. Therefore, our results indicated that Ang II stimulation facilitated the transcription of PFKP via ERK1/2 activation, which is well known to be involved in Ang IImediated cell proliferation [27]. However, the activation of ERK1/2-targeted transcription factors, such as ATF1 
a

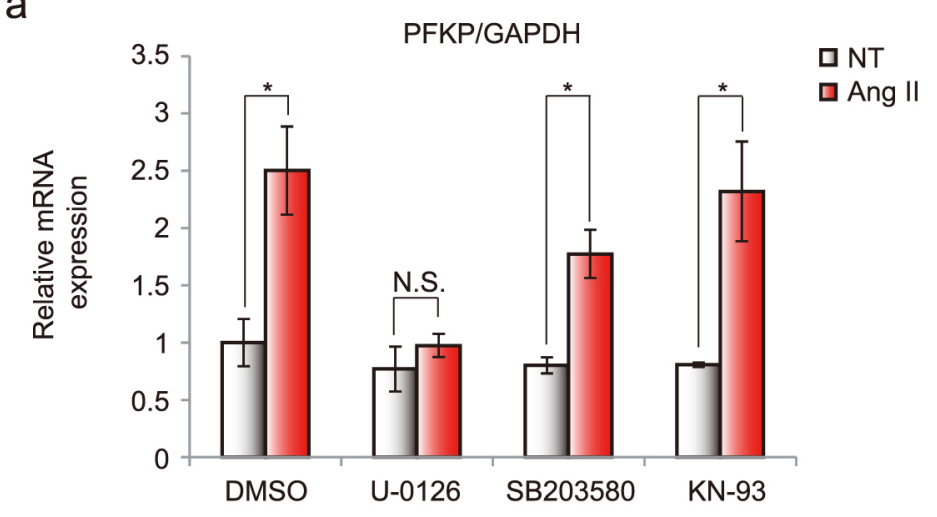

b

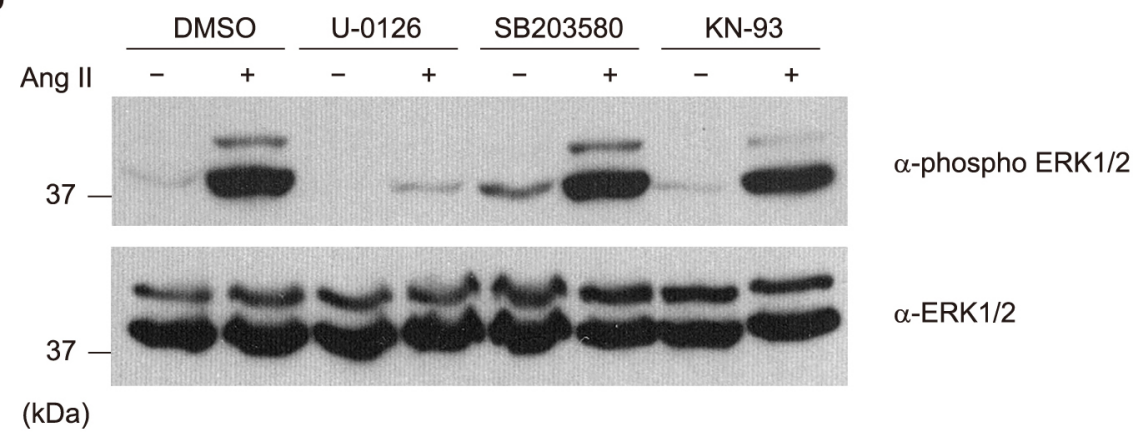

Fig. 3 The upregulation of PFKP expression by Ang II was dependent on ERK1/2 activation.

(A) MEK inhibitor U-0126 inhibited the rise of PFKP expression by Ang II. RT-qPCR was operated as in Fig. 2 (C). The cells were cultured under the condition with $10 \mu \mathrm{M} \mathrm{U}-0126,10 \mu \mathrm{M}$ SB203580 and $5 \mu \mathrm{M} \mathrm{KN}-93$ for 12 hours before $0.1 \mu \mathrm{M}$ Ang II stimulation. Data are represented as means \pm standard deviations $(n=3) . * p<0.05$. (B) U-0126 blocked the phosphorylation of ERK1/2. After treatment with Ang II for 45 minutes, the cells cultured as in (A) lysed and subjected to western blotting.

and CREM, were reported to be phosphorylated by Ang II stimulation as the fast reaction in H295R cells. In particular, the phosphorylation of these transcription factors peaked at 5 minutes from the addition of Ang II and was attenuated at 6 hours [28]. We showed that the transcriptional activation of PFKP occurred after 6 to 24 hours from Ang II treatment (Fig. 2C). Based on these findings, we presumed that the transcription of PFKP was not directly enhanced by the ERK1/2-targeted transcription factor like CREB, but other transcription factors, for example KLF4 or ZBTB7A, might mediate between ERK1/2 activation and the transactivation of PFKP.

Then, we showed that Ang II up-regulated glucose uptake in H295R cells (Fig. 4). These results indicated the possibly that Ang II stimulation raised PFKP expression, and led to facilitate glycolysis and glucose uptake. Namely, Ang II might not only induce aldosterone synthesis but also alter glucose metabolism. We speculate that Ang II may also affect the glucose metabolism of other organs that express AT1R including vascular smooth muscle, endothelium, heart, brain, kidney, and adipose tissue [27]. The relationship between aldosterone synthesis and glucose metabolism has been studied in the past. For example, cytochrome P450 oxidase CYP11A1, CYP11B1 and CYP11B2, which are enzymes that play a major role in aldosterone synthesis, are required for the reduced form of nicotinamide adenine dinucleotide phosphate (NADPH) as an electron donor [29]. Although NADPH has been known to be generated in PPP [30] and the expression of the PPP-related protein did not change in this study, the possibility remains that the induction of PFKP and enhanced glucose consumption might affect the production of NADPH. This is due to the fact that the glycolytic pathway and PPP have the common metabolites such as glucose-6-phosphate, fructose-6-phosphate and glyceraldehyde-3-phosphate [30]. Recently, epidermal growth factor receptorphosphorylated FFKP has been reported to activate phosphatidylinositol 3-kinase/Akt pathway, which results in the induction of glucose transporter 1 (GLUT1) expression [31]. Since GLUT1 is ubiquitously expressed [32], Ang II-mediated glucose uptake increase may be mediated via the FFKP-induced GLUT1 expression.

In summary, we identified PFKP as a novel target gene of Ang II by proteomic analysis. The up-regulation of PFKP was governed by ERK1/2 kinase involved in 
a

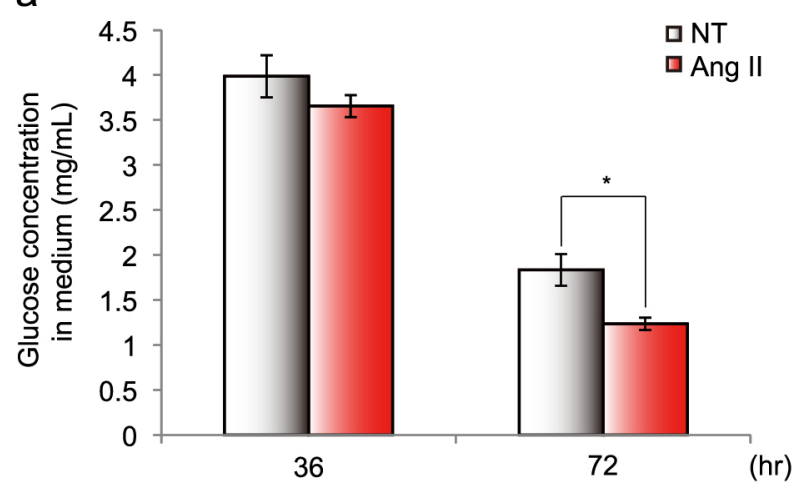

b

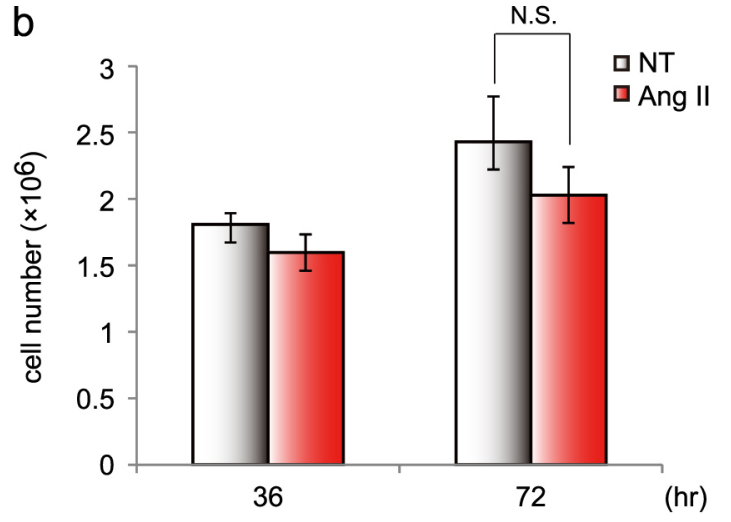

C

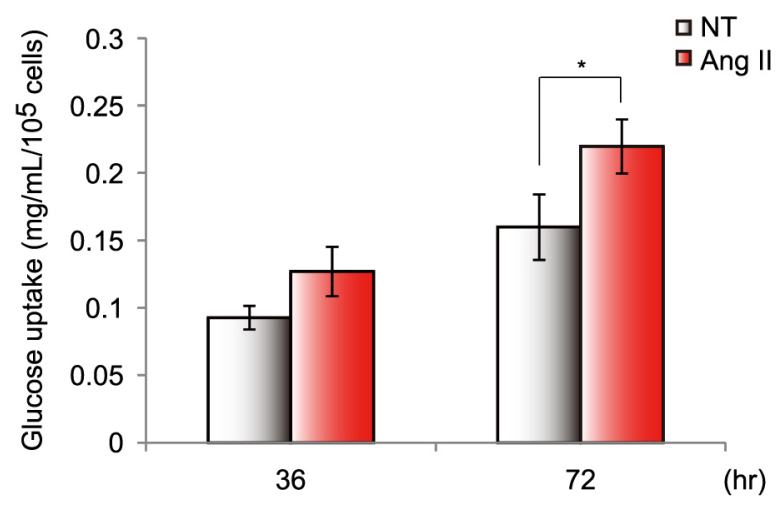

Fig. 4 Ang II increased the amount of glucose intake in H295R cells.

(A) Glucose concentrations were measured in the culture medium from the cells with/without Ang II for 36 or 72 hours. (B) Under the conditions in (A), the number of cells was counted using a hemocytometer. (C) The amount of glucose uptake per total cell number was calculated based on the results in (A) and (B). Data are represented as mean \pm standard deviation $(n=3)$. ${ }^{*} p<0.05$.

classical MAPK signaling. Furthermore, we showed that Ang II stimulation raised the amount of glucose uptake in H295R cells. This observation suggested that the treatment of cells with Ang II altered glucose metabolism in adrenal cells. Therefore, we considered that it was necessary to investigate the transition of metabolite fluxes derived from glucose stimulated by Ang II.

\section{Acknowledgments}

This work was supported by JSPS KAKENHI Grant
Numbers 26893012 (RI), 16K19550 (RI), 19K22793 (AS), the Core Research for Evolutional Science and Technology from the AMED (KI), and Platform for Drug Discovery, Informatics, and Structural Life Science from the Ministry of Education, Culture, Sports, Science and Technology, Japan (AS).

\section{Disclosure}

None of the authors have any potential conflicts of interest associated with this research.

\section{References}

1. McCormick SD, Bradshaw D (2006) Hormonal control of salt and water balance in vertebrates. Gen Comp Endocrinol 147: 3-8.

2. Romero CA, Orias M, Weir MR (2015) Novel RAAS agonists and antagonists: clinical applications and controversies. Nat Rev Endocrinol 11: 242-252.

3. Bird IM, Mason JI, Rainey WE (1998) Protein kinase A, protein kinase $\mathrm{C}$, and $\mathrm{Ca}(2+)$-regulated expression of 21 - hydroxylase cytochrome P450 in H295R human adrenocortical cells. J Clin Endocrinol Metab 83: 1592-1597.

4. Ota T, Doi M, Yamazaki F, Yarimizu D, Okada K, et al. (2014) Angiotensin II triggers expression of the adrenal gland zona glomerulosa-specific $3 \beta$-hydroxysteroid dehydrogenase isoenzyme through de novo protein synthesis of the orphan nuclear receptors NGFIB and NURR1. Mol Cell Biol 34: 3880-3894. 
5. Curnow KM, Tusie-Luna MT, Pascoe L, Natarajan R, Gu JL, et al. (1991) The product of the CYP11B2 gene is required for aldosterone biosynthesis in the human adrenal cortex. Mol Endocrinol 5: 1513-1522.

6. Lifton RP, Gharavi AG, Geller DS (2001) Molecular mechanisms of human hypertension. Cell 104: 545-556.

7. Forrester SJ, Booz GW, Sigmund CD, Coffman TM, Kawai T, et al. (2018) Angiotensin II signal transduction: an update on mechanisms of physiology and pathophysiology. Physiol Rev 98: 1627-1738.

8. Spät A, Hunyady L (2004) Control of aldosterone secretion: a model for convergence in cellular signaling pathways. Physiol Rev 84: 489-539.

9. Tian Y, Smith RD, Balla T, Catt KJ (1998) Angiotensin II activates mitogen-activated protein kinase via protein kinase $\mathrm{C}$ and Ras/Raf-1 kinase in bovine adrenal glomerulosa cells. Endocrinology 139: 1801-1809.

10. Natarajan R, Yang DC, Lanting L, Nadler JL (2002) Key role of P38 mitogen-activated protein kinase and the lipoxygenase pathway in angiotensin ii actions in H295R adrenocortical cells. Endocrine 18: 295-301.

11. Romero DG, Plonczynski M, Vergara GR, GomezSanchez EP, Gomez-Sanchez CE (2004) Angiotensin II early regulated genes in H295R human adrenocortical cells. Physiol Genomics 19: 106-116.

12. Nogueira EF, Vargas CA, Otis M, Gallo-Payet N, Bollag WB, et al. (2007) Angiotensin-II acute regulation of rapid response genes in human, bovine, and rat adrenocortical cells. J Mol Endocrinol 39: 365-374.

13. Ong SE, Blagoev B, Kratchmarova I, Kristensen DB, Steen $\mathrm{H}$, et al. (2002) Stable isotope labeling by amino acids in cell culture, SILAC, as a simple and accurate approach to expression proteomics. Mol Cell Proteomics 1: 376-386.

14. Gazdar AF, Oie HK, Shackleton CH, Chen TR, Triche TJ, et al. (1990) Establishment and characterization of a human adrenocortical carcinoma cell line that expresses multiple pathways of steroid biosynthesis. Cancer Res 50: 5488-5496.

15. Ito R, Katsura $\mathrm{S}$, Shimada $\mathrm{H}$, Tsuchiya $\mathrm{H}$, Hada $\mathrm{M}$, et al. (2014) TET3-OGT interaction increases the stability and the presence of OGT in chromatin. Genes Cells 19: 52-65.

16. Tyanova S, Temu T, Sinitcyn P, Carlson A, Hein MY, et al. (2016) The Perseus computational platform for comprehensive analysis of (prote)omics data. Nat Methods 13: 731-740.

17. Huang da W, Sherman BT, Lempicki RA (2009) Systematic and integrative analysis of large gene lists using DAVID bioinformatics resources. Nat Protoc 4: 44-57.

18. Hattangady NG, Olala LO, Bollag WB, Rainey WE (2012) Acute and chronic regulation of aldosterone production. Mol Cell Endocrinol 350: 151-162.
19. Ros S, Schulze A (2013) Balancing glycolytic flux: the role of 6-phosphofructo-2-kinase/fructose 2,6-bisphosphatases in cancer metabolism. Cancer Metab 1: 8 .

20. Webb BA, Forouhar F, Szu FE, Seetharaman J, Tong L, et al. (2015) Structures of human phosphofructokinase-1 and atomic basis of cancer-associated mutations. Nature 523: $111-114$

21. Van Schaftingen E, Jett MF, Hue L, Hers HG (1981) Control of liver 6-phosphofructokinase by fructose 2,6bisphosphate and other effectors. Proc Natl Acad Sci U S A 78: 3483-3486.

22. Suzuki J, Otsuka F, Inagaki $\mathrm{K}$, Takeda $\mathrm{M}$, Ogura $\mathrm{T}$, et al. (2004) Novel action of activin and bone morphogenetic protein in regulating aldosterone production by human adrenocortical cells. Endocrinology 145: 639-649.

23. Rainey WE, Saner K, Schimmer BP (2004) Adrenocortical cell lines. Mol Cell Endocrinol 228: 23-38.

24. Moon JS, Kim HE, Koh E, Park SH, Jin WJ, et al. (2011) Krüppel-like factor 4 (KLF4) activates the transcription of the gene for the platelet isoform of phosphofructokinase (PFKP) in breast cancer. J Biol Chem 286: 23808-23816.

25. Liu XS, Haines JE, Mehanna EK, Genet MD, Ben-Sahra I, et al. (2014) ZBTB7A acts as a tumor suppressor through the transcriptional repression of glycolysis. Genes Dev 28: 1917-1928.

26. Favata MF, Horiuchi KY, Manos EJ, Daulerio AJ, Stradley DA, et al. (1998) Identification of a novel inhibitor of mitogen-activated protein kinase kinase. $J$ Biol Chem 273: 18623-18632.

27. Forrester SJ, Booz GW, Sigmund CD, Coffman TM, Kawai T, et al. (2018) Angiotensin II signal transduction: an update on mechanisms of physiology and pathophysiology. Physiol Rev 98: 1627-1738.

28. Nogueira EF, Rainey WE (2010) Regulation of aldosterone synthase by activator transcription factor/cAMP response element-binding protein family members. Endocrinology 151: 1060-1070.

29. Rapoport R, Sklan D, Hanukoglu I (1995) Electron leakage from the adrenal-cortex mitochondrial P450Scc and P450C11 systems: NADPH and steroid dependence. Arch Biochem Biophys 317: 412-416.

30. Wamelink MM, Struys EA, Jakobs C (2008) The biochemistry, metabolism and inherited defects of the pentose phosphate pathway: a review. J Inherit Metab Dis 31: 703-717.

31. Lee JH, Liu R, Li J, Wang Y, Tan L, et al. (2018) EGFRphosphorylated platelet isoform of phosphofructokinase 1 promotes PI3K activation. Mol Cell 70: 197-210. e7.

32. Deng D, Yan N (2016) GLUT, SGLT, and SWEET: structural and mechanistic investigations of the glucose transporters. Protein Sci 25: 546-558. 